\title{
Malignant skin lesion - a case report
}

\begin{abstract}
The study was initiated from a clinical case observed during a practical field experience and an admission of a patient with advanced malignant neoplasia at a university hospital in Rio de Janeiro, Brazil. The abnormal rates of stimulants and cellular inhibitors cause a relative increase of stimulants in relation to inhibition and they advance without stopping the cellular cycle by developing cancer. Based on these concepts, it is understood that the oncological wound it's a differentiated lesion of the others, due to their cellular, proliferative and disseminated characteristics, requiring an early diagnosis, which proves the occurrence of neoplastic disease. The treatment of oncological wounds consists in minimizing the symptoms that are odor, bleeding, pruritus and pain. It becomes imperative that health professionals understand the natural history of cancer to deal appropriately with the complexities of the disease.
\end{abstract}

Keywords: nursing care, wound tumor, malignant wound, wound cancer, cancer
Volume 2 Issue 2 - 2018

\author{
Osório Evelyn Gomes,' Pereira Sandra R \\ Maciqueira, ${ }^{2}$ Reis Irlândia F Ocke ${ }^{3}$ \\ 'Nursing Specialist in Surgical Clinic, Universidade Estadual do \\ Rio de Janeiro (UERJ), Brasil \\ ${ }^{2}$ Surgical Medical Department, Universidade Estadual do Rio de \\ Janeiro (UERJ), Brasil \\ ${ }^{3}$ Coordinator of the Vascular Clinic Ocke Reis, University Gama \\ Filho, Brasil
}

Correspondence: Evelyn Gomes Osório, Nursing Specialist in Surgical Clinic, Universidade Estadual do Rio de Janeiro (UERJ), Rua Friburgo, 125, São João de Meriti, Rio de Janeiro, CEP: 25515-1 I0, Brasil,Tel +5521 991886798,

Email evelynosorioenf@gmail.com

Received: December 22, 2017 | Published: March 20, 2018

\section{Introduction}

The present study started from a clinical case of a client with malignant neoplasia in advanced stage. At the time of admission, her left breast had been removed two years before, and she had a tumor in the cervical region and a bloody mass in the axillary region, among other complications that will be detailed during this study. Aguiar described that among neoplasia patients, 5 to $10 \%$ develop cutaneous metastasis, with a chance to form oncologic ulcerations, sometimes during the last 6 months of life. The present study has become relevant because according to researches neoplastic ulcers are related to patient imprudence and their delay in seeking medical assistance, and/or late diagnosis of the professional to start treating the disease. ${ }^{1}$ This statement becomes controversial when compared to our case. The aforementioned patient has been routinely examined every year, but breast self-exam showed a nodule equivalent to the size of a bean. Initially diagnosed by hormonal imbalance, she then perceived the growth of a breast nodule and sought the health care service again, being diagnosed with carcinoma and immediately starting oncologic treatment.

The occurrence of cutaneous metastasis in this client goes against the literary reports stating that it is usually presented when there is a late diagnosis and the disease is already in advanced stages, because in this case even with the diagnosis and early interventions the skin lesion was evidenced. According to Gozzo, ${ }^{2}$ care directed to these lesions is specific and different from the guidelines found in studies in the area of wound care in general, as they aim to control symptoms rather than cure, i.e., palliative care. Realizing the difficulty of the nursing team in determining an adequate conduct to treat this wound, made us think of what would be the correct assistance approach when dressing an oncological wound. The early diagnosis of breast calcifications is essential to improve the patient survival, especially when detected in small diameters, which is possible with the technological development in the quality of the diagnostic exams, bringing expectation of cure. According to the Brazilian Ministry of Health, most in situ cancers are curable if treated before progressing to the stage of invasive cancer. At this stage (in situ), the cancer cells have not yet spread to other layers of the organ of origin. ${ }^{3}$

In situ ductal carcinoma, also known as ISDC, can progress to the invasive type (IDC), making a suitable and curable prognosis difficult. INCA's publication described that in invasive cancer the cells invade other cellular layers of the organ, go to the blood or lymphatic flow, and are capable of spreading to other parts of the body. ${ }^{3}$

We describe a case report that demonstrates the particularities in the management of neoplastic lesions and the importance of effective conduction of this care, for both the patient and the multiprofessional team.

\section{Case presentation}

Female patient, 56 years old, with malignant neoplasm of the left breast. She underwent neoadjuvant chemotherapy followed by radical left mastectomy. The histopathological report evidenced IDC grade III. Exiguous deep margin. Presence of metastatic carcinoma in 6 axillary lymph nodes. She received $60 \mathrm{~Gy}$ in 30 fractions on the left plastron (with direct electron field of $9 \mathrm{MeV}$ ), and a dose of $50 \mathrm{~Gy}$ in 25 fractions on left lymphatic drainage chains with $6 \mathrm{MV}$ photon fields. Patient progressed with left cervical and right axillary lymph node failure. She had palliative external RxT in the right axilla, with a dose of $3000 \mathrm{~Gy}$ in 10 fractions. The physical examination showed a mass in the left cervical region measuring $8.5 \times 10 \times 11 \mathrm{~cm}$, a malignant ulcerative wound in the left plastron region measuring $2 \times 2 \mathrm{~cm}$ with the presence of liquefaction necrosis, several satellite lesions with pruritus and malignant fungal ulcerated wound in the right axilla, irregular formation measuring $15 \times 11 \times 12 \mathrm{~cm}$, vegetative aspect, friable, involving dermis, epidermis and subcutaneous tissue, exudative, with bleeding in the tunneling region, odor grade II. Said lesions are formed by the infiltration of malignant cells into the skin structure causing loss of the solution of continuity of cutaneous tissue due to the uncontrolled cellular proliferation, intrinsic to the process of oncogenesis, resulting in an evolutionarily exophytic wound (Figures 1-6). 


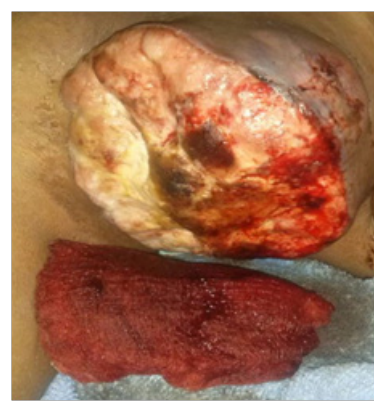

Figure I 20/03 Right Axilla

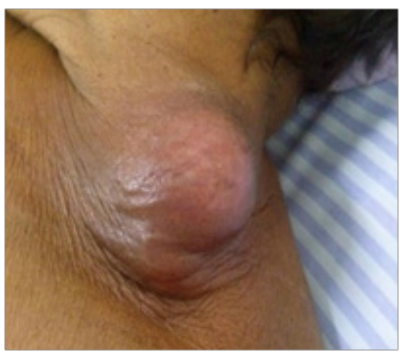

Figure 2 20/03 Cervical region, left side

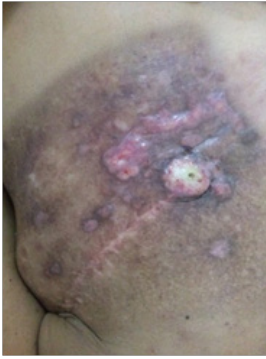

Figure 3 20/03 Plastron left side

On March 20, 2014, the patient went to the General Emergency of a university hospital located in the city of Rio de Janeiro, Brazil, showing bleeding in a right axillary tumor. She was hospitalized with SEPSIS. Advanced breast cancer, right mastectomy. The patient was admitted with fever, bleeding and pain. Laboratory tests showed leukocytosis $(16,000)$ and hypercalcemia (13.6). At this time, she was already diagnosed with bone and lung metastasis, and was undergoing clinical treatment with neoadjuvant chemotherapy and adjuvant radiotherapy and using oral antibiotic therapy with ciprofloxacin and potassium clavulanate to control the symptoms presented in right axilla ulceration without significant improvement She was hospitalized with cutaneous sepsis for parenteral antibiotic therapy. Ciprofloxacin and intravenous clindamycin hydrochloride were initiated. On March 24 of the same year, she was transferred to the Surgical Clinic for a possible intervention. During the follow-up of the patient in the hospital ward, it was possible to perceive that the tumor wound had specific characteristics differentiating it from the other wounds, mainly due to its vegetative aspect. The importance of knowing the history of the patient is clear, being it the first step to suspect of a malignant wound, and that it is a cutaneous metastasis. The nursing team of the night shift made a dressing with papain + collagenase. No possibility of palliative surgery.

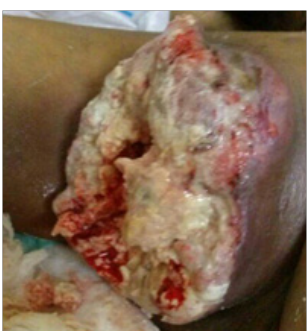

Figure $424 / 03$ Vegetative evolution in the right axilla

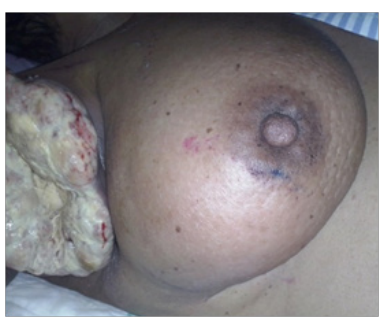

Figure 5 24/03 Vegetative evolution in the right axilla

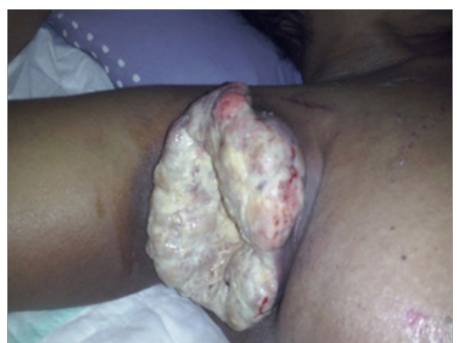

Figure 6 31/03 Improvement in wound appearance

On the following day, we could see how shocking it is to visualize a lesion that causes pain, changes the appearance, has an unpleasant odor, among other characteristics, that brings emotional discomfort even to the health care professional, not to mention those who do not have scientific knowledge like their families and people from their daily lives. It was difficult to clean and remove ointment residues. Pieces of tumor were present on the gauze of the previous dressing, and even with all the care to remove it the patient's report was of intense pain and persistent bleeding. The choice was to use cold saline to clean the wound, and the nursing team was guided not to use any other product. As of this date, we took the responsibility of changing the dressing of this patient, leaving the guidelines for the conduct to be maintained in the medical records. In this context, and considering the difficulty in handling this type of lesion properly, it was necessary to correlate the medical diagnosis with nursing care. Dealing with the dressing made it clear that such coatings were harmful, worsening the appearance and increasing the odor and bleeding. These lesions are commonly rated according to the appearance, odor and staging, as described below based on the palliative care series manual on the treatment and control of tumor wounds and pressure ulcers in advanced cancer, published by INCA. ${ }^{4}$ 
Neoplastic wounds are a subject rarely explored by the world literature when compared to other wounds, whether they are chronic or not. This lack of information generates management difficulties in the care practice and lack of standardization to make the dressings. ${ }^{5}$ The literature addresses these wounds based on the appearance they exhibit throughout their evolutionary process: ulcerative malignant lesions - when they present ulceration; ulcerated malignant fungal wounds - when they present vegetative appearance and ulcerated parts; fungal or malignant fungal wounds-when they appear cauliflowered. ${ }^{6}$ As specific protocols were used for oncological wounds and the products used were changed, the improvement of the odor was noticed, progressing to grade I and no longer noticed after a few weeks, not even when opening the dressing. The wound was cleaned with a solution of Metronidazole $0.5 \%$, and the dressing made with non-adherent coverage, achieving success in odor reduction. Pain and bleeding control are factors to be considered when choosing the ideal coverage. The aforementioned patient had a wound in the thoracic region located above the left mastectomy surgical scar (2 years ago), with purulent exudate in a small amount and the presence of pruritus reported by the patient. The second wound in the right axillary region did not have pain or odor. The reason why the relatives sought hospital care was the presence of intense hemorrhage and grade II odor. The conduct initiated by a non-specialized team using collagenase and papain as a cover was detrimental to damage control. The purpose of the dressing is to provide comfort, minimize the exudate and odor, and improve body aesthetics. Products with healing potential favor cell division, which may interfere with tumor genesis and contribute to tumor growth. Covers with debriding potential enhance the odor when they act on the devitalized tissue of the wound and should also be avoided.

\section{Discussion}

Although any type of cancer can affect the skin, the cancers most commonly associated to the formation of neoplastic wounds are breast, head and neck, kidney, lung, ovary, colon, penis, bladder cancers, lymphoma and leukemia. Among these, the breast and head and neck cancers seem to be the ones presenting the most neoplastic wound, both in their primary sites and in the metastatic sites. ${ }^{5}$ Regarding the frequency of tumor wounds, to date no studies have been carried out in the field of Brazilian oncology nursing to verify the incidence of neoplastic wounds. The basic criteria to evaluate a wound are mainly divided into identifying the wound according to the aspect, describing the characteristics of the tissue and the characteristics of the exudate, and describing the conditions of the perilesional skin. It is important to describe the wound cleanliness, and evaluate if the products/coverages are adequate for tumor lesions. As previously seen, the oncological wounds are derived from cutaneous metastasis and usually presented at the end of life, and comes to confirm the worsening of the clinical condition. Considering that tumor recurrence may be a consequence of a single altered cell, the use of cicatrizing products is questioned, since they induce cell division for tissue repair purposes, and thus, as proliferation generates regeneration, it will concomitantly promote tumor enlargement.

Patients excluded from the curative treatment phase as the clinical condition worsens progressively increase the neoplastic wound, where necrotic tissue proliferates and the use of antiseptics considered cytotoxic to the granulation tissue becomes useful because healing is not the goal. Products with healing or debriding action are contraindicated in skin tumors, even if the wound is necrotic, as it will promote the debridement and/or mitosis of tumor cells, like the aforementioned products. The ideal is a differentiated professional look for each patient in particular. Treatment will be based on each symptom presented, which are: pain control, odor control, pruritus control, and bleeding control. Regarding pain, pre-analgesia may be considered by the use of rescue dose; use of topical anesthetic around the wound; application of aluminum hydroxide directly at the site of the lesion when it is in staging; careful manipulation of the wound bed avoiding smearing; review of ongoing systemic analgesia and considering the use of antiallergic radiotherapy. ${ }^{5}$

For odor control, the use of a systemic antibiotic is recommended, and the topical application to the bed of these wounds is controversial, although it has proved useful in empirical use. Not only neoplastic wounds are controversial, but all other types of wounds, even in the case of ointments and/or antibiotic-based creams. The points of controversy concerns drug absorption, drug effectiveness, and bacterial resistance. ${ }^{5}$ Pruritus usually occurs in the initial phase of wound formation and may be due to the release of histamine inherent to the inflammatory process of the adjacent skin, either by tumor formation itself or by maceration due to the secretion drained from the tumor or by allergic processes to the products used in the dressings. (FIRMINO, 2005) The application of topical steroids such as Dexamethasone $0.1 \%$ cream is recommended to control pruritus. ${ }^{7}$

For bleeding control, it is suggested the application of a hemostatic sponge (made of collagen); care manipulation using irrigation with SF $0.9 \%$ or treated water when removing the previous dressing; use of non-adherent dressings such as satin gauzes and/or moistened with physiological solution $0.9 \%$ or treated water, as well as application of topical epinephrine directly on the bleeding sites. ${ }^{5}$ Knowing how to properly handle the tumor wound requires the nurse to know the particularities of each lesion with a differentiated look, understanding that when it comes to palliative care, the goal of the dressing is to provide relief, improve the body aesthetics, and promote patient comfort.

\section{Conflict of interest}

The author declares there are no conflicts of interest.

\section{Acknowledgements}

None.

\section{Patient consent form}

Patients permission to publish this case report was obtained and documented in a patient consent form.

\section{References}

1. Aguiar RM, Silva GRC. Os cuidados de enfermagem em feridas neoplásicas na assistência paliativa. Revista do Hospital Pedro Ernesto. 2012;11(2) 
2. Gozzo TO, Fernanda Padovani, Marceila de Andrade, et al. Ocorrência e manejo de feridas neoplásicas em mulheres com câncer de mama avançado. Escola Anna Nery. 2014;18(2):270-276.

3. INCA. ABC do câncer: abordagens básicas para o controle do câncer. Rio de Janeiro, Brasil: Ministério da Saúde; 2011. 128 p.

4. Instituto Nacional de Câncer. Tratamento e controle de feridas tumorais e úlceras por pressão no câncer avançado. Brasil: Série cuidados paliativos; 2009. 44 p.
5. Firmino F. Feridas neoplásicas: Estadiamento e controle dos sinais e sintomas. Rev Prática Hospitalar. 2005;4(42):59-62

6. Ivect $\mathrm{O}$, Lyne PA. Fungating and ulcerating malignant lesions: a review of the literature. $J$ Adv Nurs. 1990;15(1):83-88.

7. Miller C. Nursing aspects. In: Doyle D, Hanks GWC, Macdonald N. Oxford textbook of palliative medicine. J R Soc Med. 2001;97(7):356357. 